\title{
Du télé-viseur au téléspectateur
}

Quand la Télévision joue avec ceux qui la Regardent

From Television Set to Television Viewer. When Television plays with Those which Watch it

\section{Laurence Leveneur}

\section{OpenEdition \\ Journals}

Édition électronique

URL : http://journals.openedition.org/questionsdecommunication/7723

DOI : 10.4000/questionsdecommunication. 7723

ISSN : 2259-8901

\section{Éditeur}

Presses universitaires de Lorraine

\section{Édition imprimée}

Date de publication : 1 décembre 2006

Pagination : 333-348

ISBN : 978-2-86480-828-2

ISSN : $1633-5961$

\section{Référence électronique}

Laurence Leveneur, «Du télé-viseur au téléspectateur », Questions de communication [En ligne],

10 | 2006, mis en ligne le 01 décembre 2006, consulté le 11 octobre 2018. URL : http:// journals.openedition.org/questionsdecommunication/7723; DOI : 10.4000/ questionsdecommunication.7723 


\title{
$>$ NOTES DE RECHERCHE
}

\author{
LAURENCE LEVENEUR
}

Centre de recherche sur les images et les sons médiatiques Université de la Sorbonne Nouvelle Paris 3

l.leveneur@ceisme.fr

\section{DU TÉLÉ-VISEUR AU TÉLÉSPECTATEUR. QUAND LA TÉLÉVISION JOUE AVEC CEUX QUI LA REGARDENT}

Résumé. - Une récente recherche portant sur les jeux télévisés des années 70 nous a permis de mettre en évidence l'importance des formules fondées sur les dons d'observation et l'attention visuelle des téléspectateurs. Ces émissions émergent à un moment où la télévision, encore coincée entre les deux modèles médiatiques dominants que sont la radio et le cinéma, et largement traversée par des formes extra-médiatiques héritées tantôt de la littérature, tantôt du monde du spectacle, peine à développer une écriture propre. Or, certains de ces programmes, en proposant au spectateur de jouer avec les images et la télévision, questionnent la spécificité du dispositif télévisuel. L'analyse de ces jeux télévisés, en particulier ceux qui sont diffusés au moment où la question de la légitimation du média est posée de façon cruciale, montre par quels procédés la télévision met à l'épreuve l'œil du téléspectateur. Le jeu à la télévision devient alors un espace de questionnement, d'inventivité et d'expérimentation au même titre que les autres genres.

Mots clés. - Télévision, jeu télévisé, histoire, image, analyse pragmatique. 
$\square$

orsque la télévision commence à entrer dans les foyers français, elle charrie avec elle un double héritage médiatique. En effet, le cinéma et la radio restent au début des années 50 , les principaux médias de référence, sans compter l'influence notable de modèles extra-médiatiques comme le théâtre, le cabaret, le music-hall, le cirque ou encore la permanence de formes littéraires à l'image de la devinette, de l'énigme... Encore balbutiant, le nouveau-né doit répondre aux attentes multiples de spectateurs peu ou prou habitués à l'image animée : "Vu sous l'angle des croyances attachées aux images, le cinéma et la télévision sont différents, simplement parce qu'ils apparaissent dans des contextes où le savoir de l'homme sur la représentation est différent. Découvrir l'image animée quand il n'existe que de l'image fixe ou presque, produit évidemment d'autres effets que recevoir à distance de l'image animée dans un monde où celle-ci existe déjà depuis plus de trente ans » (Jost, 1998a : 82). Face à cette concurrence accrue et aux attentes de son nouveau public, la télévision doit donc tenter de résoudre, selon l'expression de Jean Thevenot (1946), la quadrature du cercle : « [...] Car la télévision s'adressera, avec les moyens de la radio, à un public qui attendra d'elle l'équivalent du cinéma » (Delavaud, 2003b: 5I). L'étude des passages intersémiotiques est un enjeu en soi : la transposition de différents genres à de nouveaux supports a d'ailleurs intéressé bon nombre de chercheurs considérant bien souvent ces opérations comme des «mouvements de déperdition » (Steimberg, 1997 : 52). Ainsi certains ont-ils pu interroger les spécificités des dispositifs cinématographiques, télévisuels et vidéo (Ellis, 1982).

Le poids de cet héritage dont la télévision peine à se délester se fait sentir jusque dans les années 70 où les critiques reprochent au nouveau médium de ne pas trouver un style qui lui soit propre avec des émissions véritablement télévisuelles. Pourtant, les chercheurs qui se sont penchés sur ces décennies ont montré que la télévision des premiers temps, bien qu'étroitement liée aux autres médias, commence à développer une écriture propre, que ce soit au travers des dramatiques en direct (Delavaud, 1998) ou de quelques programmes de variétés et d'information (Sorlin, 1997). Une récente recherche sur les jeux télévisés des années 70 conduit à une réflexion similaire (Leveneur, 2005b).

La plupart des travaux portant sur le jeu télévisé classent les émissions de ce genre à partir des catégories héritées de la réflexion du sociologue Roger Caillois et considèrent qu'il existe quatre types de jeux, ou bien fondés sur la compétition (Agôn), le hasard (Alea), le vertige (llinx) ou le travestissement (Mimicry) (Caillois, 1958). Or, l'observation d'un panorama des jeux télévisés constitué à partir des 
sources audiovisuelles de l'Inathèque de France' montre l'importance des formules sollicitant l'attention visuelle du téléspectateur'. Comment expliquer l'importance de ces émissions durant les 30 premières années d'existence de la télévision ${ }^{3}$, si ce n'est par cette quête de légitimation qui traverse ce média?

L'hypothèse est que certains jeux télévisés, fondés sur l'attention visuelle, principalement les programmes diffusés de 1950 jusqu'au début des années 80 - bien que largement influencés par des formes pré- ou extramédiatiques (Jost, 1999 : 121-123) - ont interrogé, de façon inédite, le dispositif télévisuel et, à travers lui, le regard du téléspectateur. Certains de ces programmes questionnent la « télé-vision » en tant que telle, cette vision distanciée du téléspectateur, d'ailleurs appelé « téléviseur 》 par Jean Thevenot en 1946, lorsqu'il découvre l'étrange lucarne et s'interroge sur la spécificité des conditions de réception liées à ce nouveau média (Delavaud, 2003b : 50), comme le fera quelques années plus tard John Ellis (1982) à travers la fiction. Bien que limités du point de vue de la structure ou des règles - combien de formules de jeux ont été plusieurs fois remises au goût du jour ? - les jeux télévisés ouvrent paradoxalement un espace d'inventivité et un terrain d'expérimentation. Pour commencer, nous nous intéresserons aux émissions fortement influencées par des formes pré-médiatiques qui utilisent l'image comme un référent spécifique sur lequel le téléspectateur focalise son l'attention. Ensuite, nous nous attarderons sur d'autres collections ${ }^{4}$ qui interrogent véritablement le dispositif télévisuel, et proposent aux téléspectateurs de jouer avec leurs écrans. Enfin, nous nous préoccuperons de celles qui vont au-delà de l'écran, pour dépasser l'image comme analogon et en dévoiler « l'ensemble des après et des apprêts... » (Metz, 1970 : |49).

\section{Des jeux pour capter le regard du téléspectateur : héritages pré-médiatiques}

Bon nombre de jeux télévisés des premiers temps s'inspirent largement de certaines formes extra-médiatiques, souvent littéraires, comme la devinette, l'énigme, le rébus... Ainsi Télé-Match (1954-1961) qui est

\footnotetext{
' Ce panorama est le résultat d'un travail de thèse inédit dans le cadre d'un doctorat en sciences de l'information et de la communication. Pour le détail des jeux de la décennie 1970-1979 et la méthodologie employée pour les répertorier, se référer à Leveneur (2005b).

${ }^{2}$ Ces formules représentent $14 \%$ des jeux télévisés créés et diffusés entre 1950 et 1979, contre $5 \%$ seulement pour la décennie 1980-1989.

${ }^{3}$ Nous évoquons ici l'existence « médiatique » du petit écran qui ne commence véritablement à entrer dans les foyers français qu'au cours des années 50 , bien qu'il soit officiellement né avant la Seconde Guerre mondiale.

${ }^{4}$ Nous appelons « collection » un ensemble d'émissions du même titre.
} 
considéré comme le premier grand jeu télévisé français - du moins le premier qui ait connu un succès tel que les candidats de l'émission devinrent de véritables vedettes - est-il, au départ, qualifié par ses créateurs de « jeu de l'oie télévisé » dans lequel les joueurs passent à travers cinquante-cinq décors différents correspondant aux cases du jeu de société éponyme (Télérama, 25/I0/54). De même, le célèbre « traininterlude » (Le train de la gaîté/Rébus express/Interlude, 1962-1964), diffusé au début des années 60 , ne propose-t-il pas aux téléspectateurs un rébus à résoudre ? Les transpositions de genres littéraires ou les adaptations de jeux pré-médiatiques sur un support télévisuel ont intéressé différents chercheurs (Steimberg, 1997), théorisant parfois la double relation « centrifuge » et « centripète » de la télévision à la culture et à la société (Corner, 1995). Car si la télévision se caractérise par son incroyable capacité à « ingérer » des formes culturelles qui lui sont extérieures, elle imprime aussi, sur tous les pans de la culture, ses propres images (Corner, 1995 : 5). Les émissions dont les formules s'inspirent de cet héritage pré-médiatique offrent un précieux terrain d'analyse à quiconque s'interroge sur les moyens employés par la télévision pour adapter ces formes au langage audiovisuel.

Aussi certaines émissions fondées sur les dons d'observation du téléspectateur s'inspirent-elles de formes pré-médiatiques ou radiophoniques. Elles ne questionnent pas systématiquement le rapport qui s'instaure entre le petit écran et celui qui le regarde, et soulignent, tout au plus, la façon dont la télévision des premiers temps cherche à se démarquer de ses parangons en utilisant l'image à titre d'illustration, se contentant bien souvent de faire ce que les journalistes de l'époque appellent de la radio illustrée (Leveneur, 2005b). Toutefois, certaines émissions, par leur contenu ou leur réalisation, mettent véritablement en jeu le regard du téléspectateur et les moyens dont la télévision dispose pour le captiver.

\section{La clé de l'énigme}

Le roman à énigme, forme qui atteint son heure de gloire entre les deux guerres, superpose deux récits ; celui du crime et celui de l'enquête. Le premier se caractérise par son absence, il ne peut être immédiatement présent dans le livre. Le second sert seulement de médiateur entre le lecteur et I'histoire du crime (Todorov, 197| : II-13). L'attention du lecteur est alors concentrée sur l'énigme à résoudre. En ce sens, l'enquêteur astucieux, qui tient le rôle central dans ce type de roman, procède selon Philippe Le Guern ${ }^{5}(08 / 02 / 06)$ à la manière du sémioticien : « La technique indicielle et hypothético-déductive des

${ }^{5}$ http://www.ditl.info/arttest/art3545.php 
investigations policières (Edgar Allan Poe, Emile Gaboriau, Conan Doyle) témoigne en ce sens d'un jeu herméneutique où le détective procède en sémioticien, selon le modèle de la lecture et de l'interprétation... ». Le roman à énigme est éminemment ludique, le lecteur étant amené à réfléchir, à partir des indices repérés par l'enquêteur, sur l'identité du coupable. Et l'énigme est en soi, du moins en matière de fiction, éminemment télévisuelle. En effet, elle permet de capter l'attention d'un téléspectateur souvent distrait, grâce à une narration sérielle qui constitue, selon certains analystes comme John Ellis (1982 : |26) l'un des traits caractéristiques de la télévision par rapport au cinéma.

Plusieurs jeux télévisés se fondent sur ce principe de l'énigme policière. Ainsi poursuivent-ils une tradition d'abord issue de la littérature et qui s'est ensuite développée avec la radio. Pour mémoire, nous pouvons citer Un Mystère par jour (1970-1978), jeu policier diffusé sur la deuxième chaîne et dont les auteurs et producteurs (Jean Bardin, Alain Franck et Bernard Hubrenne) venus de la radio, ont déjà participé à l'élaboration de dramatiques policières (Leveneur, 2005b : 187). Par l'intermédiaire du professeur Henri Crémieux, ils proposent aux téléspectateurs de résoudre une énigme à partir des indices donnés à l'occasion d'une courte fiction. À la même époque,TFI propose une série policière où le candidat joue le rôle d'un inspecteur chargé de mener une enquête à partir de renseignements, de témoignages, aidé par les téléspectateurs qui peuvent appeler en direct (L'inspecteur mène l'enquête, 1975-1981). Plus récemment et selon un principe associant un film et un jeu, France 3 a adapté le célèbre jeu de société Cluedo (1994-1995). Mais l'ancêtre télévisé de ces émissions requérant l'attention visuelle et la perspicacité des candidats et des téléspectateurs n'est autre que la fameuse émission de Claude Loursais : Les Cinq dernières minutes (1958-1973). Cette série met en œuvre diverses stratégies de captation du regard qu'il faut maintenant préciser. Rappelons brièvement le principe de cette dramatique policière tel que Claude Loursais l'énonçait à son lancement dans Télé-Radio 57 (1957-1958) : « Vous allez assister à un drame policier, un crime a été commis. L'inspecteur enquête, mais au moment où il va découvrir l'assassin, l'émission s'arrête. Dans un coin du studio, il y a trois ou quatre spécialistes du roman policier qui sont devant un récepteur, ils ont tout suivi attentivement, c'est vers eux que la caméra se tourne, à eux de trouver le coupable, à vous aussi bien entendu ». Comme l'émission est d'abord tournée en direct, Claude Loursais fait rejouer par les comédiens, les passages que les candidats demandent à revoir, possibilité qui n'existe d'ailleurs qu'à la télévision. Le réalisateur décide ensuite d'alterner le direct avec des séquences filmées, pour des raisons budgétaires. Les candidats disparaissent eux aussi progressivement, de telle sorte que l'émission devient une dramatique à part entière. Raymond Souplex, alias l'inspecteur Bourrel, pointait alors son doigt vers le téléspectateur en prononçant cette phrase désormais 
célèbre : «Bon Dieu, mais c'est bien sûr [...]. Rappelez-vous... », lançant ainsi les séquences cruciales grâces auxquelles chacun pouvait découvrir les indices les plus révélateurs. Créée à la fin de l'année 1957, la série est diffusée de 1958 à 1973. Après une période de transition, une deuxième version met en scène le commissaire Cabrol (Jacques Debary) et l'inspecteur Ménardeau (Marc Eyraud), de 1975 au début des années 90, remplacée ensuite par une troisième version dont les héros sont le commissaire Massard (Pierre Santini) et son adjoint Antoine Barrier (Pierre Hoden). Nous ne nous intéresserons ici qu'à la première mouture de cette collection.

Umberto Eco (1983: |4|) voyait dans la recherche d'un contact avec le public l'une des caractéristiques de la néo-télévision. La transparence énonciative de la paléo-télévision aurait disparu au profit d'une mise en scène de l'acte même d'énonciation via diverses stratégies dont le regard, face à la caméra, participe. Grâce à lui, la télévision établit un contact avec le téléspectateur. Et si les émissions de divertissement ou d'information tendaient, au début de la télévision italienne, à réduire au minimum ce contact visuel pour rendre plus crédible l'énoncé, Umberto Eco (ibid. : 148) postule un glissement progressif de ces programmes vers davantage de « simulacres d'énonciation » allant de pair avec un mélange des genres. Selon l'auteur, ce qui compte avec la néo-télévision n'est donc plus tant la véracité de l'énoncé que l'effet de vérité produit (ibid. : I48). Mais le changement de modèle relationnel postulé par Umberto Eco, repris par Francesco Casetti et Roger Odin (1990), ne résiste pas longtemps au visionnage des archives audiovisuelles françaises. Par exemple, le regard-caméra qui est l'un des procédés les plus couramment employés par les présentateurs pour établir un contact avec le téléspectateur, a été inauguré très tôt. D'ailleurs, François Jost (1998: 33) note une recommandation faite aux acteurs de la télévision par Wladimir Porché, directeur de la RTF en 1954, et allant dans ce sens. De plus, si ce regard frontal est bien un moyen employé par la télévision pour s'adresser directement à ses téléspectateurs sur le mode conversationnel, et créer par ce biais un effet d'immédiateté et d'intimité (Ellis, 1982: 133-134), il n'est pas l'apanage des genres informatifs, ni même réservé aux speakers ou aux journalistes, puisqu'on le trouve à l'œuvre dans l'émission qui nous intéresse : Les Cinq dernières minutes. À la fois jeu et fiction, ce programme, non seulement instaure avec le téléspectateur une relation d'intimité par le regard-caméra, mais encore place ce dernier dans une position d'ubiquité qui, selon André Bazin (in : Delavaud, 2003a: 54), constitue l'une des caractéristiques du « fondement psychologique du plaisir de la télévision ».

Lorsque la série devient une fiction à part entière, elle rompt avec l'une des règles du genre par l'usage quasi systématique du regard-caméra. Par ce procédé, elle instaure un rapport de proximité avec le téléspectateur, 
même lorsque celui-ci n'est plus directement sollicité par sa participation à l'émission. II reste alors un interlocuteur direct dont la sagacité, l'esprit de déduction et les capacités d'observation sont constamment mis à l'épreuve. L'émission du 3 juillet 1965 présente un cas remarquable illustrant ce rôle particulier dévolu au public. L'enquête s'interrompt vers la fin du programme, proposant au spectateur un temps de pause, un « interlude de réflexion », meublé par le mouvement de la caméra qui parcourt une pièce dans laquelle un indice est supposé trahir l'assassin. L'objectif accomplit son mouvement selon un procédé d'ocularisation interne (Jost, 1999 : 57) en imitant le mouvement d'une tête qui observerait les lieux. Ce temps de pause est signalé par un message à l'écran qui invite chacun à poser son regard sur certains détails de la pièce. Autrement dit, le regard du téléspectateur est triplement sollicité : par l'inspecteur Bourrel, tout d'abord, qui interpelle les inspecteurs en herbe lors de ce moment privilégié, par le texte même ensuite qui s'inscrit sur l'écran de télévision et ancre véritablement l'image, et par le mouvement de la caméra enfin qui reproduit celui que nos têtes seraient censées faire si nous étions véritablement sur les lieux. Le contact visuel mis au départ en œuvre par le regard face à la caméra du commissaire est comme inversé, invitant à projeter notre propre regard sur une scène illusoire. Ainsi la réalisation donne-t-elle le sentiment au téléspectateur que l'écran devient comme un prolongement de son regard. Ici, nous trouvons l'écho d'une réflexion d'André Bazin (in : Delavaud, 2003a : 54) à propos du sentiment d'ubiquité lié à l'écran de télévision et qui procure au téléspectateur un plaisir spécifique. Le critique « [...] explique que le petit écran, contrairement à l'écran de cinéma, n'est pas une surface de projection, mais le prolongement de cet organe de la vision : «la rétine d'un œil magique» qui nous découvre ce que notre œil seul ne saurait percevoir ». L'identification quasi physique de l'œil du téléspectateur à celui de la caméra permet d'établir un contact fort qui l'éloigne de la figure du « téléspectateur modèle » (Jost, 1995) qu'imaginaient certains analystes à propos de la paléo-télévision. Toutefois, cette possibilité reste limitée par la télévision elle-même qui, si elle établit avec son téléspectateur une relation de complicité forte, n'est toutefois pas en mesure de lui accorder une vision totalisante : " Hence the lack of a truly voyeuristic position for the TV viewer. It is not the TV viewer's gaze that is engaged, but his or her glance, a look without power. It is not the viewer's look that is at stake in the process, but the look of TV itself on a world beyond. There is no voyeuristic separation for the $T V$ viewer as there is for the cinema viewer $»^{6}$ (Ellis, $1982: 163$ ).

\footnotetext{
${ }^{6}$ « D'où l'absence d'une position véritablement voyeuriste du téléspectateur. Ce n'est pas le regard fixe du téléspectateur qui est engagé, mais son "coup d'œil", un regard sans pouvoir. Ce n'est pas le regard du téléspectateur qui est en jeu dans ce processus, mais celui de la télévision elle-même sur le monde qui l'entoure ». Notons à ce sujet la richesse sémantique de la langue anglaise lorsqu'il s'agit de décrire les subtilités de la vision et du regard humain (gaze, glance, look).
} 


\section{La devinette et le jeu des erreurs}

L'énigme et la devinette fonctionnent selon des procédés similaires : toutes deux associent une partie descriptive présente qui fait office d'indice et un référent absent. En outre, elles fonctionnent sur ce qui est caché : il s'agit de trouver l'identité de l'assassin ou la nature d'un référent. Ainsi Oscar Steimberg (1997:57) définit-il la devinette comme un « jeu avec réponse cachée », et la différencie-t-il de l'énigme « dont le point de départ est similaire, mais dans laquelle on accentue le rôle du destin, de la divinité ou des grands déterminismes sociaux dans une réponse aux dimensions tragiques $\gg$. La forme la plus répandue de la devinette à la télévision est le jeu de questions-réponses ou quiz show. Mais la devinette peut parfois s'appuyer sur des documents visuels, comme dans Le Schmilblic (1969-1987), où les référents qu'il s'agit de reconnaître sont en général fragmentés ou montrés sous un angle particulier qui limite les possibilités du regard spectatoriel. Présentée par Guy Lux, cette émission s'appuie principalement sur des images fixes. Elle s'apparente aisément à ce que les journalistes de l'époque qualifient de radio illustrée, puisqu'elle permet au téléspectateur, après avoir bien observé l'image en question, de vaquer ensuite à ses occupations tout en écoutant les différents concurrents émettre leurs hypothèses sur la nature de l'objet. Rien d'étonnant à cela puisque la formule est inspirée d'un jeu radiophonique (Leveneur, 2005b : 187). Et même lorsque l'image sur laquelle porte la devinette est télévisuelle (séquence d'une émission, reportage, etc.), elle est souvent au service d'une forme de réflexivité télévisuelle qui vise à fidéliser l'audience en l'interrogeant sur les programmes d'une chaîne (Télé-Fidélité, TFI, 1975-1976).

Il faudra attendre le début des années 80 pour que la télévision, tout en s'appuyant sur cette forme traditionnelle qu'est la devinette, réussisse à capter le regard du téléspectateur grâce à une expérience des plus spectaculaires décrite par Jean-Marc Vernier (1987) dans un article particulièrement éclairant. L'auteur y analyse la célèbre émission intitulée La Chasse aux trésors, diffusée sur Antenne 2 en 1981, réalisée par Guy Job et co-présentée par Philippe Gildas et Philippe de Dieuleveult. II y observe le renversement de la prééminence, jusque-là sans conteste, de la parole sur l'image télévisuelle. Le dispositif de l'émission, qui permet au téléspectateur de voir des images auxquelles les candidats en plateau n'ont pas accès, le fait ainsi bénéficier d'un savoir supplémentaire et tirer de ce « plus d'image » un plaisir spécifique lié à la sensation d'ubiquité et au pouvoir qui lui est alors donné. Dès lors, le spectateur est rendu plus attentif par cette «fascination ludique »: "L'intelligence de ce dispositif, c'est de dépasser la diffusion élémentaire d'images-à-voir pour réaliser une véritable programmation du regard » (Vernier, 1987 : 42). Par ce procédé novateur, l'émission, qui oblige les candidats à résoudre trois « énigmes »-qui, si l'on s'en tient aux distinctions opérées par 
Oscar Steimberg, se rapprochent davantage de la devinette - oriente le regard du téléspectateur. Ce « téléguidage » fascinant est autrement mis en œuvre dans des émissions également fondées sur l'attention visuelle, mais héritières d'une autre forme de jeu extra-médiatique : le jeu des erreurs.

Le principe du jeu des erreurs glissées dans un document visuel est connu. II a fait la part belle de certaines pages « jeux » de la presseécrite. II a été repris dans des émissions liées au thème de la sécurité routière. Citons par exemple La Bonne conduite (1976-1982), une émission présentée par Manuelle Lidsky et Michel Vidaud, puis par Michel Denisot. Réalisée par Guy Saguez, elle propose aux jeunes téléspectateurs de TFI quelques tests sur le code de la route, notamment grâce à un petit film dans lequel les concurrents doivent repérer des erreurs de conduite ou des anomalies. L'émission use et abuse des images : dans le générique d'abord, qui se présente comme une mosaïque d'images, dans les jingles ensuite qui annoncent les différentes séquences du jeu et qui sont en fait des dessins accompagnés de bruitages amusants, et pour le jeu principal enfin qui est une séquence filmée. Plus, l'image y est mise en abîme puisque le colonel Lagache, chargé d'indiquer aux téléspectateurs les erreurs qu'il fallait repérer, se tourne pour cela vers un grand écran dont il se sert comme d'un tableau de professeur. Afin d'attirer l'attention du téléspectateur, l'émission joue avec les cadres et les cercles pour mettre en valeur les panneaux routiers importants ou signaler les erreurs commises par les personnages du court métrage.

La permanence de formes simples comme la devinette ou l'énigme dans les jeux télévisés des premiers temps n'empêche nullement l'innovation langagière. Au contraire, elle permet d'observer la façon dont le média naissant a su adapter ces genres et les recycler « en les passant au moule de son langage audiovisuel » (Lochard, Boyer, 1995 : 100). Mais au-delà de l'image analogique, les émissions qui nous intéressent ont aussi initié, de façon ludique, un questionnement sur le dispositif télévisuel.

\section{Jover avec l'écran du télé-viseur}

Selon les défenseurs du paradigme hérité d'Umberto Eco, l'introduction du processus d'interactivité est considérée comme un autre élément novateur typique de la néo-télévision. Ainsi les jeux télévisés diffusés au début des années 90, comme Hugo Délires (1992-1993) - qui deviendra, en 1993, Les Délires d'Hugo (1993-1994) - ou encore Télétennis (1994), étonnent-ils la presse par leur caractère innovant. En effet, ces émissions permettent aux jeunes téléspectateurs de jouer à des jeux vidéo, à distance, via leur combiné téléphonique. Or, jusque-là, la participation des 
téléspectateurs à distance consistait à aider les candidats en plateau, par téléphone, courrier ou minitel, à répondre directement à des questions, et en usant des mêmes biais.

Encore une fois, les archives audiovisuelles révèlent la précocité du média. Très tôt, la télévision a proposé à ses jeunes téléspectateurs de jouer avec leur écran, notamment grâce à un jeu de décodage qui leur permettait, à partir d'une grille découpée dans un journal de programme, de trouver le message secret qui leur était adressé par la télévision (Jeu de la grille, 1953). Dès la fin des années 60, l'ORTF propose à son public une émission intégralement fondée sur un principe interactif, intitulée L'Arbalète de Noël (1969-1970). Ce programme quotidien d'une quinzaine de minutes, présenté par Guy Lux et réalisé par Jean Cohen, permet aux téléspectateurs, alors munis d'un téléphone, et ayant renvoyé aux producteurs une carte-réponse trouvée dans une Caisse du Crédit Agricole, de diriger à distance le tir d'une arbalète. Pour cela, un viseur adapté à l'objectif d'une caméra est dirigé par un caméraman dont on a pris soin de bander les yeux. Ainsi doit-il suivre les indications données par des concurrents au téléphone qui jouent pour 3 millions d'anciens francs. Ici, l'écran de télévision prend donc la forme d'un véritable dispositif ludique.

Néanmoins, on peut s'interroger sur le plaisir qu'en tire le téléspectateur non participant, celui qui est condamné à observer passivement dans son fauteuil les candidats diriger le tir de l'arbalète. D'ailleurs, le caractère éphémère de l'émission renseigne, en partie, sur les limites de son succès. Mais, ce qui n'a pas fonctionné à la fin des années 60 a pourtant fait chavirer le cœur des téléspectateurs de France 3, en 1992, avec Hugodélires. Alors même que certains journalistes s'interrogent sur la non-participation du téléspectateur qui n'est pas candidat et se trouve « réduit à regarder, par dessus l'épaule de l'heureux élu du jour, la même partie sans surprises » (Le Leurch, Télérama, 03/03/96), le petit personnage du nom de Hugo réunit, à 20 heures, plus de six millions de téléspectateurs. Peut-être trouve-t-on l'explication de ce succès dans la fascination qu'éprouve alors le jeune spectateur, également amateur de jeux vidéos, à observer les performances des autres joueurs en plateau ou au téléphone, selon un principe d'identification qui participe de ce que Roger Caillois (1985: 66-67) nomme la mimicry: « $[\ldots]$ l'identification au champion, à elle seule, constitue une mimicry parente de celle qui fait que le lecteur se reconnaît dans le héros du roman, le spectateur dans le héros du film. [...] Dans ces conditions, outre le spectacle, prend naissance, au sein du public, une compétition par mimicry, qui double l'agôn véritable du terrain ou de la piste ».

Mais cette « ludicisation » du dispositif télévisuel reste exceptionnelle. Jusqu'au début des années 80 , la plupart des formules de jeux 
d'observation ou d'attention visuelle se fondent sur les formes simples évoquées précédemment. II faut attendre le mois d'août 1980 pour qu'émerge, grâce au travail du Service de recherche de l'ORTF, un jeu questionnant véritablement l'image télévisuelle, au-delà de son aspect analogique et selon une formule qui s'est développée indépendamment des formes pré- ou extra-médiatiques.

\section{Des jeux pour disséquer le regard du téléspectateur : au-delà de l'écran, l'image}

Le 24 août 1980, I'Institut national de l'audiovisuel (INA) et le célèbre producteur Jean Frapat proposent aux téléspectateurs de FR3 deux séries d'émissions diffusées le dimanche soir à 21 heures et intitulées Télétests? Cette émission fait suite à une première série d'« expériences 》 diffusées, en 1972, sous le titre La Boîte à malices. Ce jeu programmé sur la première chaîne, et réalisé par Jean-Pierre Barizien, est successivement présenté par Dominique Paturel et Christian Marin, puis par Georges de Caunes et André Naudin. Son principe est simple : il s'agit d'inviter deux familles de téléspectateurs à venir jouer à la télévision, en leur faisant endosser le rôle de speakerines, de cameramen, de bruiteurs et autres métiers liés au petit écran, afin de susciter par ce biais de véritables rencontres entre le public et les professionnels du média. Jean-Christophe Averty participe à la dernière émission, diffusée le 30 septembre 1973, afin d'y présenter ses trucages les plus spectaculaires, avec le concours de son collaborateur habituel Max Debrenne. Parmi les jeux proposés aux familles invitées, il en est un qui nous intéresse plus particulièrement. II s'agit de montrer aux candidats un petit film monté à l'envers et de les interroger ensuite sur le sens qu'ils donnent à cette séquence. Autrement dit, ce jeu sans concours ni récompense, propose de questionner les mécanismes d'interprétation suscités par une image. Quelques années plus tard, Jean Frapat reprend cette idée de tests liés à l'image et aux sons télévisuels et la développe dans la série Télétests, miroir de la télévision et de ses effets sur les téléspectateurs.

\section{Une véritable expérience de laboratoire}

Claude Villers (Télétests, 24/08/80), débauché de France-Inter pour animer cette collection, présente ainsi le principe de l'émission : « Les enfants préfèrent jouer avec les boîtes, les emballages. Nous, chez nous, il y a toujours une belle boîte qui se trouve dans un coin du salon... ça

\footnotetext{
${ }^{7}$ Une première série comporte six émissions (diffusées du 24/08/80 au 28/09/80), et une deuxième, dix émissions (diffusées du 04/I0/8I au 06/I2/8I).
} 
s'appelle la télévision. Et nous nous sommes dit :"pourquoi ne pas jouer avec cette grosse boite? Pourquoi nous ne profiterions pas de toutes ces images que l'on nous distribue à longueur de journées pour essayer d'en faire des petits jeux?" ». Ces tests proposés aux téléspectateurs et aux candidats du jeu font appel à leur mémoire visuelle, à leur capacité d'observation ou à leur perspicacité. Réalisée par Michel Hermant, l'émission propose, de façon réflexive, un regard sur la télévision et sur sa capacité à créer une opinion. Tel est le principe d'un test intitulé « La rumeur » dans lequel un candidat, après avoir regardé un reportage, le raconte à un second, qui lui-même transmet ce récit à un troisième, et ainsi de suite jusqu'au dernier joueur, qui rapporte à la fin une histoire n'ayant plus grand chose à voir avec le contenu initial du reportage. Ce qui permet d'observer, selon les termes de Jean Frapat (Télé Journal, 23/08/80), « comment tout ce que les gens ont dans la tête (stéréotypes, préjugés ou préoccupations personnelles) interfère et brouille leur lecture des images $\gg$. Pour ce producteur, la télévision reste encore au début des années 80 « une courroie de transmission entre le cinéma, le music hall et le public » (ibid.). Aussi propose-t-il aux spectateurs de « mettre la télévision en question » et d'exploiter les «formidables ressources » (ibid.) de ce jeune média.

Pour cela, il met en place un dispositif qui s'inscrit dans la lignée des expériences de laboratoire du Service de la recherche de I'ORTF et du travail de Pierre Schaeffer (Soulez, 2005). Lors de la première émission, les candidats sont enfermés dans une cage octogonale à demi-transparente qu'une caméra située au-dessus du plateau filme en plongée. À chaque interlude, Claude Villers explique au téléspectateur, qui devient un interlocuteur privilégié, le principe du prochain test en voix over tandis que les candidats prennent place dans le décor. Pour rendre cette expérience plus « scientifique », le producteur a sélectionné un panel varié de « cobayes » d'âges et d'origines sociales différents. Afin de comparer les réactions des « téléspectateurs des villes et les téléspectateurs des champs » (émission du |8/|0/8|), une émission entière est ainsi consacrée aux agriculteurs. Une autre envisage de confronter la sagacité de ceux qui ont grandi avec la télévision et de ceux qui l'ont vu naître (émission du 31/08/80), ou encore celle des « couples naturels » (frères, sœurs, jumeaux) et des « couples artificiels 》 (maris-femmes, fiancés). Toutefois, cette « radioscopie » du téléspectateur se déroule dans une ambiance conviviale qui n'a rien à voir avec celle des vrais laboratoires et qui fait de l'émission un piège amusant digne de la Caméra invisible (1964-1971, Antenne 2).

\section{"Réactiver le regard du spectateur »}

L'émission fait mouche et pour certains critiques de télévision, elle provoque le téléspectateur, triture son imagination et constitue une « passionnante expérience de métamorphose du regard [...] mais aussi [une] première tentative intelligente de réveil du téléspectateur 
ensommeillé que nous sommes tous devenus au fil des ans, assommés par la surabondance d'images » (Frapat, Le Progrès, 07/09/80). Loin de la radio imagée ou du cinéma, par sa réalisation même, l'émission met en jeu le regard du spectateur de télévision. Pendant que les tests animés et commentés par Claude Villers questionnent les pouvoirs de l'image et du son, Michel Hermant guide subtilement le regard du téléspectateur vers les endroits normalement cachés du plateau de télévision, déjouant ainsi l'illusion de la transparence et du simulacre télévisuel. Ainsi la réalisation de Télétests dépasse-t-elle le rôle habituellement dévolu à l'image télévisuelle et évoqué par John Ellis (1982: 129-130) à propos de la très sérieuse BBC, par exemple : « Sound tends to anchor meaning on TV, where the image tends to anchor it with cinema. [...] Especially in British broadcast TV (possibly most hide-hound in the world), the image is to be kept literal for almost all the time. There are licensed exceptions (science-fiction, rock music programmes) where experimentation with the physical composition of the video image can take place, but the rule is that the image must show whatever is before the camera with the minimum of fuss and conscious technique $\Perp^{8}$. La réalisation de Michel Hermant dévoile les projecteurs, les caméras et autres matériels audiovisuels placés sur le décor, mettant non seulement à l'honneur les métiers et techniques de la télévision comme le faisait déjà l'équipe de $L a$ Boîte à malices, constituant une forme de réflexivité destinée à rappeler que nous sommes bien assis devant un écran de télévision. De la même façon, le montage est nettement mis en avant par différents procédés de fondu enchaîné, de mise en cadre, de sonorisation, aussi bien au niveau méta-discursif qu'au niveau audiovisuel. Claude Villers se transforme progressivement en professeur, décodant avec le téléspectateur le langage télévisé. II est à la fois l'hôte bienveillant qui met à l'aise les candidats et un guide privilégié. Le montage le fait apparaitre comme un narrateur visible qui commente les images des « cobayes » en train de subir les différents tests ou attire le regard vers les images qui sont en train d'être disséquées. Le regard du téléspectateur est également sollicité par différents jeux de focalisation. II est d'abord un joueur potentiel qui possède les mêmes informations que les candidats en plateau, ce qui le place du point de vue cognitif dans une situation de focalisation interne : « L'environnement cognitif du téléspectateur est le même que celui qui se trouve dans l'écran [...] quand il prend connaissance des informations, fictives ou réelles, en même temps que lui » (Jost, 1999 : 57). Puis, une fois la surprise du jeu dépassée, son savoir est supérieur à celui du joueur en plateau et il évolue

\footnotetext{
${ }^{8}$ « Le son tend à ancrer le sens à la télévision, quand c'est l'image qui tient ce rôle au cinéma. [...]. À la télévision britannique notamment (et peut-être de façon plus beaucoup plus répandue dans le monde), l'image doit rester littérale la plupart du temps. II existe quelques exceptions admises (la science-fiction, les émissions de musique consacrées au rock) où les expérimentations avec la composition physique des images vidéo peut avoir sa place, mais la règle est que l'image doit montrer tout ce qui se trouve devant la caméra sans que la dimension technique ne soit mise en avant ou consciente ».
} 
vers une situation de focalisation spectatorielle : « Le téléspectateur est alors "omniscient" : il est partout [...], il sait des choses que ne sait pas [...] le candidat » (ibid. : 57 ). Cette situation lui permet de diriger son attention non plus sur le jeu lui-même ou sur le contenu des images télévisées, mais plutôt sur les candidats dont il peut désormais analyser les réactions. Mais, à l'évidence, ce décodage du langage télévisé, qui passe notamment par un montage serré de l'émission - le producteur ne garde, en effet, sur les soixante heures d'enregistrement que six heures au total pour les émissions de la première série (Jampolsky, Télérama, 20/08/80) est rapidement perçu par les journalistes comme la marque d'une maitrise technique qui va de paire avec la fin de la spontanéité des candidats. On regrette ainsi l'ère du direct. Le jeu de Jean Frapat est rapidement critiqué pour son manque d'intérêt, lié non seulement à l'absence de gain, mais aussi à ses intentions savantes (Cressole, Libération, 23-24/08/80). Néanmoins, l'émission reste une expérience des plus rares car elle interroge, de façon ludique, les mécanismes de l'image télévisuelle et le regard du téléspectateur.

\section{Conclusion}

L'originalité et la force visuelle de certaines émissions comme Les raisins verts (1963-1964) de Jean-Christophe Averty restent des exceptions et la télévision naissante semble plutôt développer un art de la parole, fondé sur ce qu'André Bazin (in :Delavaud, 2003a :55) considère comme étant une donnée essentielle de la « réception télévisuelle » : l'art de l'intimité. Peut-être le média est-il alors trop jeune pour développer un langage propre, ou peut-être le téléspectateur lui-même est-il trop frileux face à ces innovations : « La télévision a vécu entre la routine et la découverte. Vingt ans ne lui ont pas suffi pour se créer un langage original d'autant que les téléspectateurs, vite habitués à "leurs" programmes, ont résisté à l'aventure » (Sorlin, 1997 :235).

La majeure partie des émissions fondées sur l'attention visuelle jouent davantage sur la fidélisation du public ou la mise en avant des technologies liées au médium, notamment durant les années 70 lorsque les jeux télévisés évoluent vers des formules plus figées (Leveneur, 2005b). Ici, les dispositifs de captation du regard décrits furent pourtant autant de tentatives questionnant les spécificités du médium par rapport à ses parangons médiatiques. Ces programmes ont esquissé une réflexion sur le dispositif télévisuel et sur la manière dont le téléspectateur peut se l'approprier. Ils nous permettent de découvrir le jeu télévisé sous une facette qui n'est plus celle de l'industrie télévisuelle, du recyclage des formules à succès ou du spectacle télévisé, mais plutôt sous l'angle d'une école du regard. 
À la fin des années 80 , à l'aube de la privatisation des chaînes et de l'internationalisation des formats de jeux télévisés, les émissions fondées sur les capacités d'observation des candidats s'amenuisent au profit de formats plus courts et/ou spectaculaires. L'on peut donc s'étonner que la chaîne Arte choisisse de diffuser, depuis le 4 février 2006, un jeu qui vise à exercer la mémoire visuelle des candidats et des téléspectateurs. Mémoquiz est le premier jeu programmé chaque samedi à 18h30 sur la chaîne franco-allemande. II est présenté comme « un jeu accessible à tous car il porte sur les images et sur le sens de l'observation... \» (site de la chaîne Arte, 21/0 //06). Un site internet y sera même associé pour permettre à chacun d'y puiser des références mais surtout d'y trouver « une grille de jeu qui permet de participer en direct pour s'évaluer ». Mais, la chaîne Arte n'en est pas à son coup d'essai en la matière, puisque Claire Doutriaux, réalisatrice d'une émission intitulée Karambolage, vient de recevoir le Grimme Preis pour la conception et l'idée de cette courte séquence diffusée chaque dimanche soir à 20 heures depuis le 4 janvier 2004, et dont l'un des principes n'est autre qu'une devinette portant sur un plan télévisé dont il s'agit de déterminer le lieu de tournage ! Surprenante répétition de l'histoire pour une télévision qui n'en a visiblement pas fini de se poser des questions avec les images.

\section{Références}

Abdesselem C., 1980, « Jean Frapat : "Il faut réinventer la télévision" », Télé Journal, 23 août.

Caillois R., 1958, Les jeux et les hommes, Paris, Gallimard, 1991.

Casetti Fr., Odin R., 1990, « De la paléo- à la néo-télévision. Approche sémiopragmatique », Communications, 51, pp. 9-26.

Corner J., 1995, Television form and public address, London, Edward Arnold.

Cressole M., 1980, « Un nouveau jeu, pas de jeu. Télétests », Libération, 23-24 août.

Delavaud G., 1998, «Dramaturgie du télévisuel. Le Rouge et le Noir, une dramatique de Pierre Cardinal », pp. 133-150, in : Bourdon J., Jost F., dirs, Penser la télévision. Actes du colloque de Cerisy, Paris, Nathan/INA.

— 2003a, « André Bazin, critique de télévision », pp. 47-56, in : Bourdon J., Frodon J.-M., dirs, L'œil critique. Le journaliste critique de télévision, Paris, INA/De Boeck.

- dir., 2003b, « Un siècle de télévision. Anticipation, utopie, prospective », Dossiers de l'audiovisuel, I 12.

Eco U., 1985, La guerre du faux, trad. de l'italien par M. Tanant avec la collab. de P. Caracciolo, Paris, Grasset.

\footnotetext{
${ }^{9} \mathrm{http}: / /$ www.artetv.com/fr/semaine/244,broadcastingNum=529522, day = I, week=6,year= 2006.html.
} 
Ellis J., 1982, Visible Fictions. Cinema, television, video, London, New York, Routledge.

Favre M., 1994, Les Cinq dernières minutes, monographie d'une série policière de la télévision française (1958-1973), Mémoire de DEA de sciences politiques, IEP, Paris.

Heitz B., 1980, « Interview de Jean Frapat. Télétests », Le Progrès, 7 sept.

Jampolsky M., 1980, « Six jeux pour retrouver l'appétit de la télévision », Télérama, 20 août.

Jost Fr., 1995, « Téléspectateurs modèles et modèles de téléspectateurs », pp. 49-62, in : Esquenazi J.-P., dir., La télévision et ses téléspectateurs, Paris, Éd. L'Harmattan.

- 1998a, Le temps d'un regard. Du spectateur aux images, Paris, Éd. Méridiens Klincksieck.

— 1998b, « Quand y a-t-il énonciation télévisuelle ? », pp. 29-58, in : Bourdon J., Jost Fr., dirs, Penser la télévision. Actes du colloque de Cerisy, Nathan, INA.

- 1999, Introduction à l'analyse de la télévision, Paris, Éd. Ellipses.

Le Guern P., « L'énigme policière », site internet du Dictionnaire International des termes littéraires, http://www.ditl.info/arttest/art3545.php, consulté le 08/02/06.

Le Leurch V., 1993, « Victorieux Hugo », Télérama, 3 mars.

Leveneur L., 2005a, « Jouer à regarder la télévision. Le téléspectateur à l'épreuve de l'image: Télétests », Les figures du spectateur, Paris, université de la Sorbonne Nouvelle, pp. 8I-84.

— 2005b, « La fabrique du jeu. De l'artisanat à l'âge d'usine », pp. |8|-209, in : Jost Fr., dir., Année 70. La télévision en jeu, Paris, Éd. du CNRS.

Lochard G., Boyer H., 1995, Notre écran quotidien. Une radiographie du télévisuel, Paris, Dunod.

Metz C., 1970, Essais sur la signification au cinéma, Paris, Klincksieck, 2003.

Sorlin P., 1997, « Recherche d'une écriture », pp. 235-237, in : Bourdon J., Chauveau A., Denel F., Gervereau L., Méadel C., dirs., La grande aventure du petit écran. La télévision française 1935-1975, Paris, BDIC/INA.

Soulez G., 2005, « Parole captée, parole capturée : de Pierre Schaeffer aux années 70 », pp. 107-129, in : Jost Fr., dir., Années 70. La télévision en jeu, Paris, Éd. du CNRS.

Steimberg O., 1997, « Des genres populaires à la télévision: étude d'une transposition », Réseaux, 81, pp. 51-62.

Loursais Cl., 1957-58, Télé-Radio 57, 688, déc.-janv.

Thevenot J., 1946, L'âge de la télévision et l'avenir de la radio, Paris, Les Éditions ouvrières.

Todorov T., 1971, « Typologie du roman policier », pp. 9-19, in : Poétique de la prose, 1980.

Vernier J.-M., 1987, « Du jeu au spectacle télévisé ludique. L'exemple de la "Chasse aux trésors" », Quaderni, 2, pp. 27-43. 